\title{
Hydrogen-rich saline improves non-alcoholic fatty liver disease by alleviating oxidative stress and activating hepatic PPAR $\alpha$ and PPAR $\gamma$
}

\author{
XIAO ZHAI ${ }^{1,2 *}$, XIAO CHEN $^{2 *}$, JIANCAN LU ${ }^{3}$, YAPING ZHANG ${ }^{3}$, \\ XUEJUN SUN ${ }^{4}$, QIN HUANG $^{3}$ and QIJIN WANG ${ }^{3}$ \\ ${ }^{1}$ Graduate Management Unit; Departments of ${ }^{2}$ Orthopedics and ${ }^{3}$ Endocrinology, Changhai Hospital; \\ ${ }^{4}$ Faculty of Naval Medicine, Second Military Medical University, Shanghai 200433, P.R. China
}

Received November 26, 2015; Accepted January 5, 2016

DOI: $10.3892 / \mathrm{mmr} .2017 .6120$

\begin{abstract}
Non-alcoholic fatty liver disease (NAFLD) comprises a range of liver diseases, between steatosis and non-alcoholic steatohepatitis and liver cirrhosis, which are closely associated with diabetes mellitus. Previous studies have indicated that oxidative stress is a key factor in the development of NAFLD. Molecular hydrogen $\left(\mathrm{H}_{2}\right)$ may ameliorate oxidative stress injuries by selectively neutralizing peroxynitrite and hydroxyl radicals. The present study evaluated the effects of $\mathrm{H}_{2}$ on NAFLD in rats and concluded that $\mathrm{H}_{2}$-rich saline had significant therapeutic effects on NAFLD induced by hyperglycemia and hyperlipidemia, as demonstrated by hematoxylin and eosin and terminal deoxynucleotidyl-transferase-mediated dUTP nick end labeling staining. $\mathrm{H}_{2}$-rich saline improved fasting blood glucose, fasting insulin, insulin sensitivity and glucose tolerance, and lowered the expression levels of tumor necrosis factor alpha, interleukin-1 beta, 3-nitrotyrosine and 8-hydroxy-2'-deoxyguanosine in the liver. In addition, the present study revealed that $\mathrm{H}_{2}$-rich saline could significantly increase peroxisome proliferator-activated receptor (PPAR) $\alpha$ and PPAR $\gamma$ expression in hepatocytes. In conclusion, $\mathrm{H}_{2}$-rich saline may significantly improve NAFLD, possibly by reducing oxidative stress and activating hepatic PPAR $\alpha$ and PPAR $\gamma$ expression.
\end{abstract}

Correspondence to: Dr Qijin Wang or Dr Qin Huang, Department of Endocrinology, Changhai Hospital, Second Military Medical University, 168 Changhai Road, Shanghai 200433, P.R. China

E-mail: wangqijin8668601@163.com

E-mail: qxinyi1220@163.com

*Contributed equally

Key words: non-alcoholic fatty liver disease, oxidative stress, inflammation, hydrogen, peroxisome proliferator-activated receptor

\section{Introduction}

Non-alcoholic fatty liver disease (NAFLD) represents a range of liver diseases, between steatosis and non-alcoholic steatohepatitis and liver cirrhosis (1), and is considered a public health problem (2). Evidence suggests that NAFLD is more common in patients with diabetes compared with the general population by approximately threefold (3). Prospective studies have revealed that diabetes mellitus (DM) is an independent risk factor for NAFLD development and liver-associated mortality $(4,5)$.

A number of mechanisms are shared between NAFLD and DM, including insulin resistance, hyperinsulinemia, and increased oxidative stress and inflammation. Among them, oxidative stress and inflammation serve an important role (6). In DM with insulin resistance, increased lipolysis occurs, generating more free fatty acids (FFAs). FFAs may be oxidized in the mitochondria, and increased FFA oxidation leads to the generation of more reactive oxygen species (ROS), which can trigger oxidative stress injuries. Therefore, the study and development of novel antioxidant drugs to neutralize free radicals and to reduce their biomolecular effects may provide potential options for the prevention and treatment of NAFLD.

Molecular hydrogen $\left(\mathrm{H}_{2}\right)$ is a colorless, odorless and tasteless diatomic gas. It has previously been reported that $\mathrm{H}_{2}$ could ameliorate oxidative stress injuries by selectively scavenging peroxynitrite $\left(\mathrm{ONOO}^{-}\right)$and hydroxyl radicals $(\bullet \mathrm{OH})$, the two most cytotoxic ROS (7). The therapeutic effects of $\mathrm{H}_{2}$ have been demonstrated in ischemia-reperfusion injuries $(8,9)$, transplantation injuries (10) and other injuries associated with oxidative stress. $\mathrm{H}_{2}$-rich saline has been widely studied in atherosclerosis, stress-induced nerve damage, type 2 DM (T2DM), cisplatin-induced renal injury and Parkinson's disease (11-14).

In 2011, Kamimura et al (15) reported that $\mathrm{H}_{2}$ was beneficial for obesity, DM and energy metabolism in mice. Considering the roles of oxidative stress in NAFLD injury, the present study hypothesized that, as a specific free radical scavenger, $\mathrm{H}_{2}$ could improve NAFLD induced by a high-sugar and high-fat diet, and evaluated the effects of $\mathrm{H}_{2}$ on NAFLD as well as the possible underlying mechanisms. 


\section{Materials and methods}

Ethics statement. All animal experimental protocols were approved by the ethics committee of Changhai Hospital affiliated to the Second Military Medical University (Shanghai, China) and were conducted in accordance with their guidelines.

Animals and grouping. A total of 24 male Sprague-Dawley rats (age, 10 weeks; weight, $250-280 \mathrm{~g}$ ) were purchased from the Shanghai Laboratory Animal Center of the Chinese Academy of Sciences (Shanghai, China). Rats were housed at $24 \pm 2^{\circ} \mathrm{C}$ under a 12 -h light/dark cycle, and fed ad libitum. All rats received humane care according to the Guide for the Care and Use of Laboratory Animals (16). The animals were randomly divided into three groups: i) The control group $(\mathrm{n}=8)$, which was fed a normal diet $(15 \% \mathrm{kcal}$ from fat), and normal saline $(5 \mathrm{ml} / \mathrm{kg})$ was administered intraperitoneally twice daily; ii) the model group $(\mathrm{n}=8)$, which was fed a high-sugar and high-fat diet, and normal saline $(5 \mathrm{ml} / \mathrm{kg})$ was administered intraperitoneally twice daily; and iii) the $\mathrm{H}_{2}$-rich saline treatment group $(\mathrm{n}=8)$, which was fed a high-sugar and high-fat diet, and $\mathrm{H}_{2}$-rich saline $(5 \mathrm{ml} / \mathrm{kg})$ was administered intraperitoneally twice daily. The high-fat diet was prepared according to Li et al (17) and contained 2\% cholesterol, 7\% lard, $8.3 \%$ yolk, $16.7 \%$ sucrose and $66 \%$ standard diet, which provided $4.66 \mathrm{kcal} / \mathrm{g}$ with an energy composition of $31.59 \%$ from fat, $51.73 \%$ from carbohydrate and $16.68 \%$ from protein.

Animal model establishment. Lipotoxicity was induced by a high-fat diet, and streptozotocin (STZ; Sigma-Aldrich; Merck Millipore, Darmstadt, Germany) was injected to cause glucotoxicity, which subsequently led to islet $\beta$-cell failure and apoptosis (18). Briefly, a single injection of STZ $(25 \mathrm{mg} / \mathrm{kg})$ was administered via the tail vein in $0.1 \mathrm{~mol} / 1$ citrate buffer (pH 4.2) followed by a continuous high-fat and high-sugar diet for 8 weeks. Subsequently, an oral glucose tolerance test was performed as described previously (19). Rats from the control and treated groups received $2 \mathrm{~g} / \mathrm{kg}$ glucose orally; a $2 \mathrm{~h}$ blood glucose result measuring $>1.2 \mathrm{mmol} / 1$ or a random blood glucose result measuring $>16.8 \mathrm{mmol} / \mathrm{l}$ in rats was considered successful model establishment.

$\mathrm{H}_{2}$-rich saline preparation. $\mathrm{H}_{2}$-rich saline was prepared as previously described (16). Briefly, $\mathrm{H}_{2}$ gas was dissolved in normal saline for $2 \mathrm{~h}$ under $0.4 \mathrm{MPa}$ pressure to saturation. The concentration was measured with gas chromatography to ensure the hydrogen level was $>0.6 \mathrm{mmol} . \mathrm{H}_{2}$-rich saline was prepared each week and stored at $4{ }^{\circ} \mathrm{C}$ in aluminum bags until ready for use.

Determination of serum biochemical markers, and insulin sensitivity and resistance. Blood was collected from the tail vein at 8 weeks, and serum alanine transferase (ALT), total bilirubin (TBIL), total cholesterol (TC), triglycerides (TG), fasting blood glucose (FBG) and fasting insulin (FINS) levels were determined using a biochemistry analyzer. In addition, the insulin sensitivity index (ISI) and homeostasis model assessment-insulin resistance (HOMA-IR) were calculated as follows: HOMA-IR = (fasting blood glucose $\mathrm{x}$ fasting insulin) $/ 22.5 ;$ ISI $=1$ (fasting blood glucose $\mathrm{x}$ fasting insulin) .
Insulin tolerance tests were conducted on the three rat groups as described previously (20).

Hematoxylin and eosin $(H \& E)$ staining. After 8 weeks, the rats were anesthetized using by an intraperitoneal injection of $10 \%$ aqueous solution $(0.3 \mathrm{ml} / 100 \mathrm{~g})$ of chloral hydrate (Huai'an Xingzhi Biological Technology Co., Ltd., Huaian, China) and their livers were harvested, sectioned, fixed in $4 \%$ paraformaldehyde and embedded in paraffin. The paraffin blocks were then sliced into $4 \mu \mathrm{m}$ sections and deparaffinized according to Shi et al (21).

Sections were stained with H\&E and images were captured using a light microscope. A pathologist that was blinded to the animal groups evaluated the slides and scored each liver tissue specimen for steatosis, inflammation and fibrosis based on the criteria proposed by previous studies (22-24). Briefly, for steatosis: Score 0, none present; score 1, steatosis $<33 \%$ of the parenchyma; score 2 , steatosis $34-66 \%$ of the parenchyma; score 3 , steatosis $>67 \%$ of the parenchyma. For inflammation: Score 0 , no foci of inflammation; score $1,<1$ foci per two 200x fields; score 2, one foci per two 200x fields to one foci per one 200x field; score 3, one to two foci per one 200x field; score 4, $>2$ foci per one 200x field. For fibrosis: Score 1, zone-3 perisinusoidal fibrosis; score 2, zone-3 perisinusoidal fibrosis with portal fibrosis; score 3 , zone-3 perisinusoidal fibrosis and portal fibrosis with bridging fibrosis; and score 4, cirrhosis. The total score (steatosis + inflammation + fibrosis) of each rat was calculated and the average score for each group was determined.

Terminal deoxynucleotidyl-transferase-mediated dUTP nick end labeling (TUNEL) staining. TUNEL staining was performed using an In Situ Cell Death Detection kit (Roche Diagnostics, Basel, Switzerland). Liver sections were heated to $60^{\circ} \mathrm{C}$ for dewaxing and were then rehydrated according to standard protocols and as previously reported (21). After cooling to room temperature, the sections were incubated with $20 \mu \mathrm{g} / \mathrm{ml}$ proteinase $\mathrm{K}$ solution for 20 min followed by incubation in TUNEL reaction mixture for $1 \mathrm{~h}$ at $37^{\circ} \mathrm{C}$. Converter-alkaline phosphatase antibody was then added to the samples for $1 \mathrm{~h}$ at $37^{\circ} \mathrm{C}$. Subsequently, sections were washed with PBS three times for $5 \mathrm{~min}$, followed by color development in the dark with nitroblue tetrazolium and 5-bromo-4-chloro-3-indolylphosphate. Ten fields were randomly chosen (x200). The number of apoptotic hepatocytes was counted in each field and an average was calculated.

Determination of tumor necrosis factor alpha $(T N F-\alpha)$ and interleukin-1 beta $(I L-1 \beta)$. Rat livers were collected and washed in normal saline, and were then homogenized immediately on ice in $1 \mathrm{ml}$ normal saline at $2-8^{\circ} \mathrm{C}$. The homogenates were centrifuged at $3,000 \times g$ at $4^{\circ} \mathrm{C}$ for $15 \mathrm{~min}$. The expression levels of TNF- $\alpha$ (catalog no. H052) and IL-1 $\beta$ (catalog no. H002) were measured using commercial ELISA kits (Nanjing Jianchen Bioengineering Institute, Nanjing, China) according to the manufacturer's protocol.

Determination of caspase-3 (CASP-3) activity. Similar methods were used to determine CASP-3 activity as previously described (21). Livers were harvested at 8 weeks, and 
CASP-3 activity was determined with the CASP-3/CPP32 Fluorometric Assay kit (BioVision, Inc., Milpitas, CA, USA). Briefly, hepatocyte lysates were incubated with $10 \mu \mathrm{g} / \mathrm{ml}$ proteinase $\mathrm{K}$ for $30 \mathrm{~min}$ at $37^{\circ} \mathrm{C}$ in a 96 -well plate. The peptide substrate DEVD-AFC $(5 \mu \mathrm{l})$ was then added to initiate the reaction. After incubation in the dark at $37^{\circ} \mathrm{C}$, a fluorometer was used to read the plate using a 400-nm excitation filter and a 505-nm emission filter. Fold increase in CASP-3 activity was calculated by comparing with the level of the control.

Determination of 3-nitrotyrosine (3-NT) and 8-hydroxy-2'-deoxyguanine (8-OHdG). Similar methods were employed as previously reported (25). Liver sections were deparaffinized in xylene, rehydrated with ethanol and pretreated with $10 \mu \mathrm{g} / \mathrm{ml}$ proteinase $\mathrm{K}$ for $30 \mathrm{~min}$ at $37^{\circ} \mathrm{C}$. Subsequently, the sections were incubated in $10 \%$ bovine serum albumin (Nanjing Jianchen Bioengineering Institute) for $20 \mathrm{~min}$. After overnight incubation at room temperature with anti-8-OHdG (1:200; catalog no. ZY-1131R) and anti-3-NT antibodies (1:200; catalog no. 28-60252P) from Heyzer Ye Biological Technology Co., Ltd. (Shanghai, China). An alkaline phosphatase-conjugated secondary antibody (1:500; catalog no. E030210; Shanghai Yanhua Bio-Tech Co., Ltd., Shanghai, China) were added for $1 \mathrm{~h}$ at $37^{\circ} \mathrm{C}$, and the sections were incubated with diaminobenzidine. The number of 8-OHdG- and 3-NT-positive cells were counted under a light microscope, and integral optical densities were calculated with Image-Pro Plus software version 6 (Media Cybernetics, Inc., Rockville, MD, USA).

The concentrations of $3-\mathrm{NT}$ and $8-\mathrm{OHdG}$ in the liver were determined using commercial ELISA kits [catalog nos. JK-(a)-5053 and JK-(a)-1571; Shanghai Jinkang Medicine Technology Co., Ltd., Shanghai, China], according to the manufacturer's protocol. Liver tissues were homogenized in $2 \mathrm{ml} 10 \mathrm{mM}$ phosphate-buffered saline ( $\mathrm{pH}$ 7.4). After centrifugation at $10,000 \times \mathrm{g}$ for $30 \mathrm{~min}$ at $37^{\circ} \mathrm{C}$, the levels of 3-NT and 8-OHdG in the supernatant were measured using the corresponding kits.

Determination of the expression levels of peroxisome proliferator-activated receptor $(P P A R) \alpha$ and PPAR $\gamma$. The protein expression levels of PPAR $\alpha$ and PPAR $\gamma$ in the liver were examined using immunohistochemistry. The transcription of PPAR $\alpha$ and PPAR $\gamma$ were determined by reverse transcription-quantitative polymerase chain reaction (RT-qPCR). Total RNA was extracted using TRIzol ${ }^{\circledR}$ reagent according to the manufacturer's protocol (Invitrogen; Thermo Fisher Scientific, Inc., Waltham, MA, USA). The RT reaction was performed using the Superscript II Reverse Transcriptase kit (Invitrogen; Thermo Fisher Scientific, Inc.) in a $20 \mu \mathrm{l}$ volume with $1 \mu \mathrm{g}$ total RNA; RT was conducted at $16^{\circ} \mathrm{C}$ for $30 \mathrm{~min}, 42^{\circ} \mathrm{C}$ for $42 \mathrm{~min}$ and $85^{\circ} \mathrm{C}$ for $5 \mathrm{~min}$. The RT product ( $1 \mu \mathrm{l} \mathrm{cDNA}$, corresponding to $6.25 \mathrm{ng}$ RNA) was used to conduct subsequent qPCR analyses in an ABI Prism 7300 Sequence Detection system (Applied Biosystems; Thermo Fisher Scientific, Inc.), with the SYBR ${ }^{\circledR}$ Green PCR kit (Applied Biosystems; Thermo Fisher Scientific, Inc.). Each experiment was performed in duplicate for each gene under the following cycling conditions: Initial template denaturation at $95^{\circ} \mathrm{C}$ for $5 \mathrm{~min}$, followed by 40 cycles at $95^{\circ} \mathrm{C}$ for $10 \mathrm{sec}, 60^{\circ} \mathrm{C}$ for $20 \mathrm{sec}, 72^{\circ} \mathrm{C}$ for $20 \mathrm{sec}$ and $78^{\circ} \mathrm{C}$ for $20 \mathrm{sec}$, and a final $10 \mathrm{~min}$ extension step at $72^{\circ} \mathrm{C}$.
The primer sequences were as follows: PPAR $\alpha$, forward 5'-GGT CTT AAC CGG CCC-3', reverse 5'-AAA CGC AAC GTA GAG-3'; PPAR $\gamma$, forward 5'-CTG TTT TAT GCT GTT ATG GGT GAA A-3', and reverse 5'-GCA CCA TGC TCT GGG TCA A-3'; and GAPDH, forward 5'-TGG GTG TGA ACC ACG AGA A-3', and reverse 5'-GGC ATG GAC TGT GGT CAT GA-3'. The $\Delta \Delta \mathrm{Cq}$ method was used to to normalize mRNA expression levels to those of GAPDH (26).

Statistical analysis. All data are presented as the mean \pm standard deviation $(n=8)$ and were analyzed with SPSS 9.1 software (SPSS, Inc., Chicago, IL, USA). Differences between groups were compared with one-way analysis of variance followed by the Student-Newman-Keuls post hoc test. Glucose tolerance and insulin tolerance test results were analyzed by mixed model for repeatedly measured data for multiple comparisons. $\mathrm{P}<0.05$ was considered to indicate a statistically significant difference.

\section{Results}

$H \& E$ staining of the liver. H\&E staining was used to observe liver morphology by microscopy (Fig. 1). As presented in Fig. 1A, rats in the control group demonstrated a normal architecture with hepatocytes arranged in plates aligned to sinusoids converging to centrilobular veins. Rats in the model group, which were fed a high-sugar and high-fat diet, presented with moderate to severe steatosis, ballooning degeneration, piecemeal necrosis and inflammatory cell infiltration. In addition, early fibrosis was detected. In the $\mathrm{H}_{2}$ group, a relatively normal histologic architecture was observed, with little steatosis, ballooning degeneration, necrosis and inflammatory cell infiltration. The average histopathological scores are presented in Fig. 1C. Scores in the model group were significantly higher than those in the control and $\mathrm{H}_{2}$ groups ( $\mathrm{P}<0.05$ model group vs. control and $\mathrm{H}_{2}$ groups).

TUNEL staining. For analysis of apoptosis, TUNEL staining results are presented in Fig. 1B. At a magnification of x200, the nuclei of hepatocytes were clearly stained. Some occasional TUNEL-positive cells were detected in the control group. Significantly more apoptotic cells were present in the model group, in which the apoptotic cells were significantly shrunken and the nuclei were strongly stained. In the $\mathrm{H}_{2}$ group, the number of apoptotic cells was significantly smaller than that in the model group (Fig. 1D; $\mathrm{P}<0.05$ model group vs. control and $\mathrm{H}_{2}$ groups).

Serum biochemical markers. To assess lipid metabolism and liver function, serum levels of ALT, TBIL, TC and TG were measured at 8 weeks. ALT and TBIL levels in the model group were slightly higher compared with the control and $\mathrm{H}_{2}$ groups (Fig. 2A and B); however, the differences were not statistically significant $(\mathrm{P}=0.08)$. The TC and TG levels in the model group were significantly higher compared with those in the control and $\mathrm{H}_{2}$ groups (Fig. 2C and $\mathrm{D} ; \mathrm{P}<0.05$ model group vs. control and $\mathrm{H}_{2}$ groups).

At 8 weeks, the serum FBG and FINS levels were also examined to assess the function of pancreas islets (Fig. 2E and F). For FBG, the differences between the model group and the control 


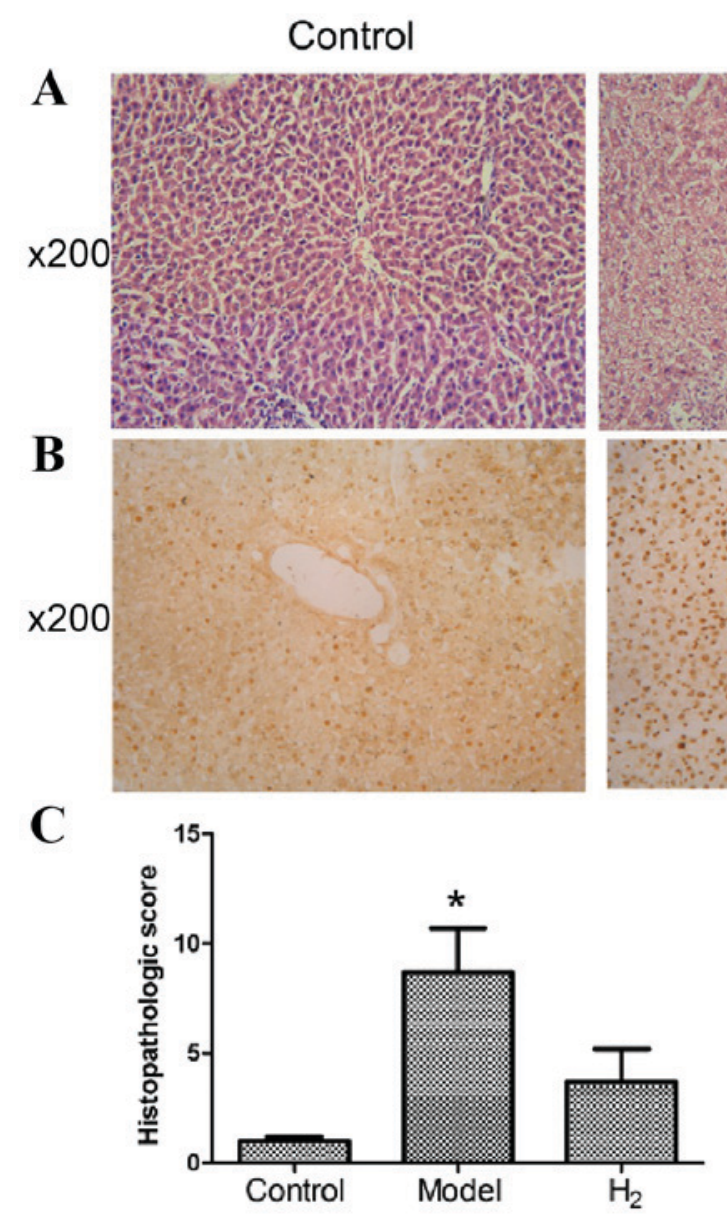

Model
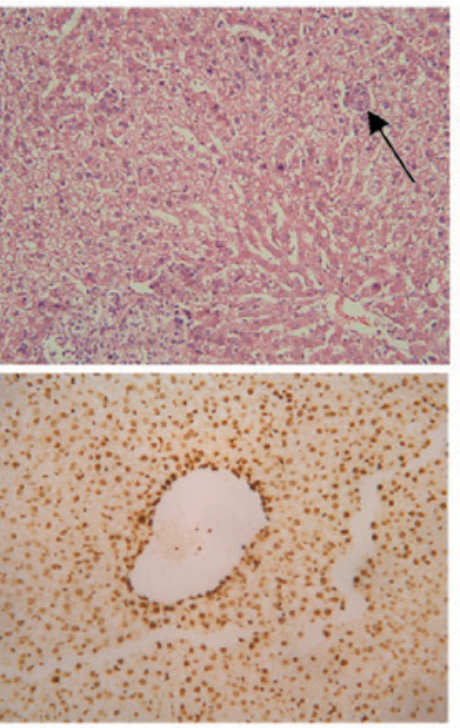

D
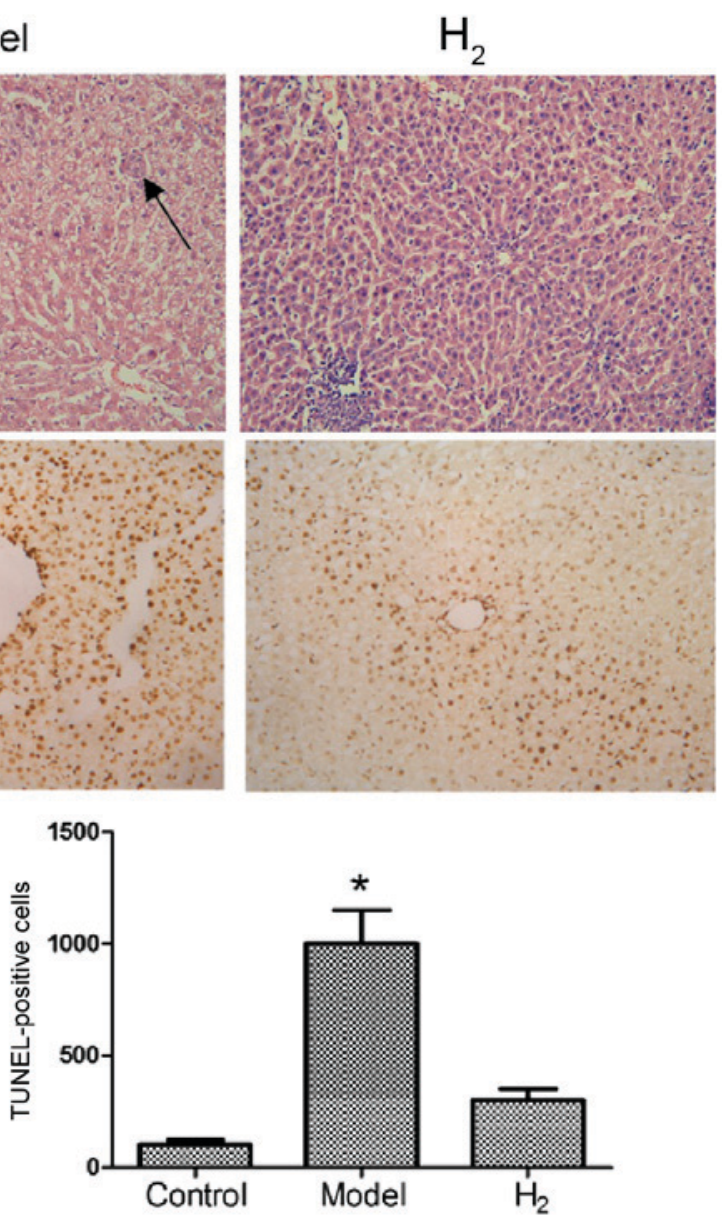

Figure 1. Histopathological findings and TUNEL staining. (A) Control group demonstrated a normal architecture with hepatocytes arranged in plates aligned to sinusoids converging to centrilobular veins. The model group presented with moderate to severe steatosis, ballooning degeneration, piecemeal necrosis and inflammatory cell infiltration, as indicated by the arrow. Early fibrosis was also detected. $\mathrm{The}_{2} \mathrm{H}_{2}$ group revealed a relatively normal histologic architecture with little steatosis, ballooning degeneration, necrosis and inflammatory cell infiltration. (B) The number of TUNEL-positive cells (brown) was significantly greater in the model group compared with in the $\mathrm{H}_{2}$ group. (C) Histopathologic score correlated with the hematoxylin and eosin staining results. "P<0.05 vs. control and $\mathrm{H}_{2}$ groups. (D) Apoptotic cell counting indicating that $\mathrm{H}_{2}$-rich saline significantly decreased the percentage of TUNEL-positive cells compared with the model group. ${ }^{2} \mathrm{P}<0.05$ vs. control and $\mathrm{H}_{2}$ groups. $\mathrm{H}_{2}$, molecular hydrogen; TUNEL, terminal deoxynucleotidyl-transferase-mediated dUTP nick end labeling.

or $\mathrm{H}_{2}$ groups were not statistically significant; for FINS, the difference was statistically significant $(\mathrm{P}<0.05$ model group vs. control and $\mathrm{H}_{2}$ groups).

Finally, the ISI and HOMA-IR were calculated to assess insulin resistance, and it was revealed that in the $\mathrm{H}_{2}$ group, when compared to the model group, the ISI was significantly elevated and the HOMA-IR was significantly reduced $(\mathrm{P}<0.05$ $\mathrm{H}_{2}$ vs. model group).

Insulin sensitivity and glucose tolerance tests. At 8 weeks, the insulin tolerance test and glucose tolerance test were performed. The insulin tolerance test was performed to assess pituitary function and adrenal function, and as presented in Fig. 2I, following injection of insulin serum glucose levels gradually decreased over time. The rate of decrease in the model group was significantly lower than that in the other two groups. At 60 and $90 \mathrm{~min}$ after the injection, the difference was statistically significant $\left(\mathrm{P}<0.05\right.$ model group vs. control and $\mathrm{H}_{2}$ groups, $\mathrm{P}<0.01$ model group vs. control and $\mathrm{H}_{2}$ groups, respectively). The glucose tolerance test was performed to diagnose diabetes, insulin resistance and impaired beta cell function. As presented in Fig. 2J, following glucose intake blood glucose gradually increased, reached a peak at $30 \mathrm{~min}$ and subsequently decreased ( $\mathrm{P}<0.05$ model vs. control and $\mathrm{H}_{2}$ groups).

Determination of 3-NT and 8-OHdG in liver cells. 3-NT, a biochemical marker of peroxynitrite, and $8-\mathrm{OHdG}$, a product of free radical oxidative damage to DNA, have been reported to be ameliorated by $\mathrm{H}_{2}(25)$. The number of 3-NT- and 8-OHdG-positive cells were detected and are presented in Fig. 3A-D. The model group had more 3-NT-positive cells (Fig. 3A and C) and more 8-OHdG-positive cells (Fig. 3B and D) compared with the control and $\mathrm{H}_{2}$ groups $(\mathrm{P}<0.05$ model group vs. control and $\mathrm{H}_{2}$ groups).

TNF- $\alpha, I L-1 \beta$ and CASP-3 in the liver. The levels of the inflammatory cytokines TNF- $\alpha$ (Fig. 3E) and IL-1 $\beta$ (Fig. 3F) in the model group were significantly greater compared with the control and $\mathrm{H}_{2}$ groups. CASP-3 activity in the model group was significantly greater compared with the control and $\mathrm{H}_{2}$ groups (Fig. 3G; $\mathrm{P}<0.05$ model group vs. control and $\mathrm{H}_{2}$ groups).

PPAR $\alpha$ and PPAR $\gamma$ expression in the liver. PPAR $\alpha$ and PPAR $\gamma$ protein expression was detected using immunohistochemistry 

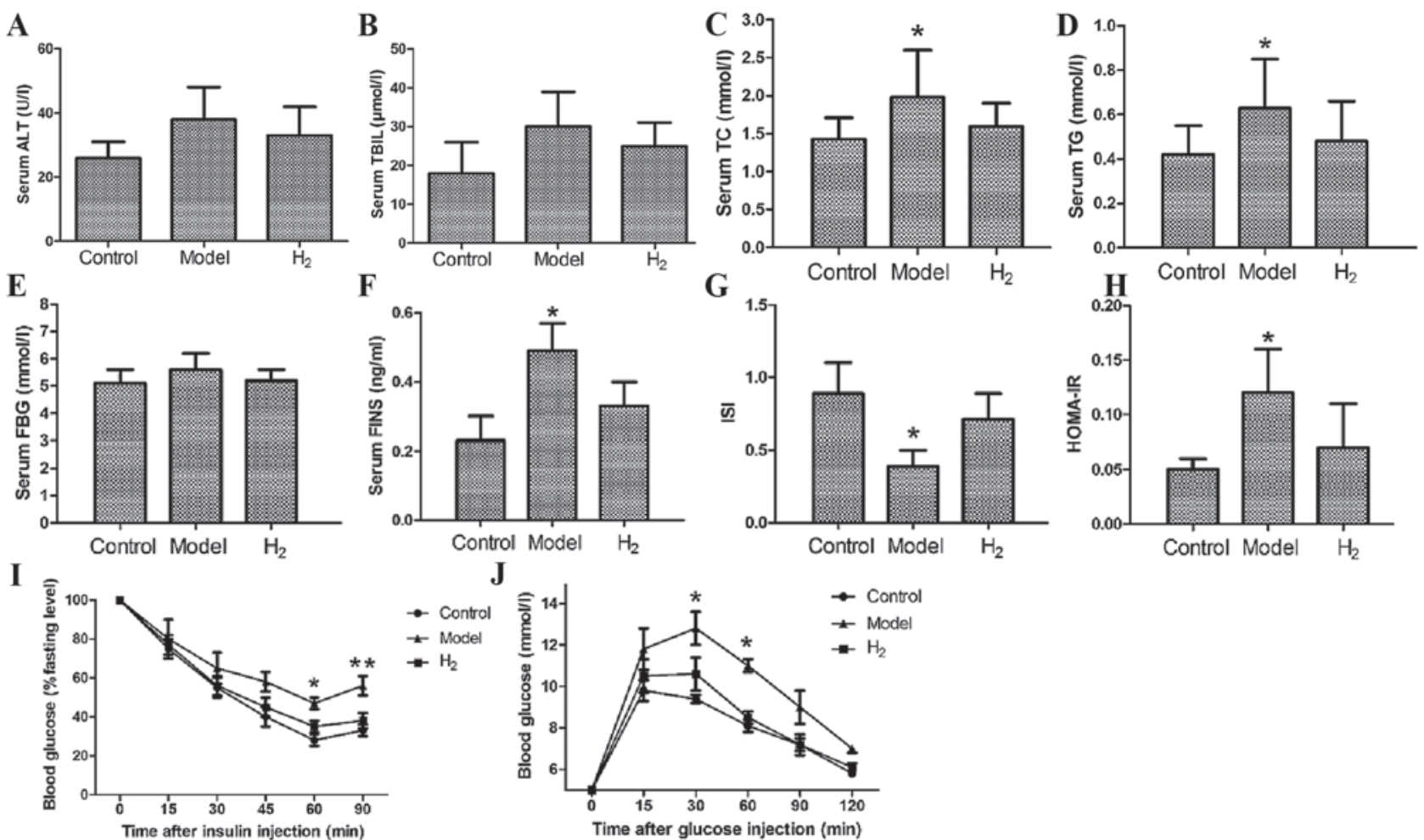

Figure 2. Biochemical marker analysis, insulin tolerance and glucose tolerance tests. (A) Serum ALT and (B) TBIL levels were higher in the model group than in the control and $\mathrm{H}_{2}$ groups. The difference was not statistically significant. $\mathrm{H}_{2}$ significantly decreased serum levels of (C) TC and (D) TG compared with the model group. Serum levels of (E) FBG and (F) FINS. (G) Results of the ISI and HOMA-IR measurements. (I) Insulin tolerance test; after insulin administration, blood glucose gradually decreased. (J) Glucose tolerance test; after glucose intake, blood glucose gradually increased and reached its peak at 30 min and then decreased. ${ }^{*} \mathrm{P}<0.05$ vs. control and $\mathrm{H}_{2}$ groups; ${ }^{* *} \mathrm{P}<0.01$ vs. control and $\mathrm{H}_{2}$ groups. ALT, alanine transferase; FBG, fasting blood glucose; FINS, fasting insulin; $\mathrm{H}_{2}$, molecular hydrogen; HOMA-IR, homeostasis model assessment-insulin resistance; ISI, insulin sensitivity index; TBIL, total bilirubin; TC, total cholesterol; TG, triglycerides.

(Fig. 4A-D). As shown in Fig 4A and C, PPAR $\alpha$ protein expression was enhanced in the $\mathrm{H}_{2}$ group compared with the control and model groups. As shown in Fig. 4B and D, PPAR $\gamma$ protein expression was also increased in the $\mathrm{H}_{2}$ group compared with the control and model groups. These differences were statistically significant. The immunohistochemical results were confirmed by RT-qPCR (Fig. 4E and F; P<0.05 model vs. $\mathrm{H}_{2}$ group).

\section{Discussion}

The present study demonstrated that $\mathrm{H}_{2}$-rich saline significantly ameliorated NAFLD, as demonstrated by reduced serum ALT, TBIL, TC, TG, FBG and FINS levels, improved insulin sensitivity and glucose tolerance, and reduced hepatocyte apoptosis, inflammation and oxidative stress. The mechanism may possibly function by upregulating the expression of PPAR $\alpha$ and PPAR $\gamma$.

The key pathophysiological process associated with NAFLD is insulin resistance, which is common in T2DM. Insulin resistance within adipocytes causes lipolysis, which subsequently results in the generation of excess FFAs that are released into circulation and finally delivered to the liver. When the import and synthesis of FFAs exceeds the capacity of the liver to use them, hepatic steatosis occurs. Excessive FFAs are metabolized within the mitochondria, peroxisomes and microsomal system, which leads to lipid peroxidation, production of excess ROS and initiation of the inflammatory response. At present, the 'two-hit' theory of steatohepatitis pathogenesis, proposed by Day and James in 1998, is widely accepted (27). The theory separates the pathogenesis into two 'hits'. The first hit is lipid accumulation due to insulin resistance and hyperlipidemia, which leaves the hepatocytes more vulnerable to injuries. Oxidative stress and the inflammatory response serve an important role in the second hit, which directly leads to hepatocyte injury. Therefore, NAFLD is a downstream effect of oxidative stress (28). Reduction of the overproduced ROS may theoretically improve NAFLD damage.

As an antioxidant, $\mathrm{H}_{2}$ has been reported to scavenge $\bullet \mathrm{OH}$ and $\mathrm{ONOO}^{-}$, the two most toxic ROS in cells. In addition, $\mathrm{H}_{2}$ protects organs from tissue damage caused by severe oxidative stress induced by inflammation, intense exercise, cardiac infarction, cessation of blood flow and organ transplantation, among others (17). $\mathrm{H}_{2}$ is highly flammable, and it is not safe to preserve and use $\mathrm{H}_{2}$ for clinical practice and laboratory experiments. Therefore, $\mathrm{H}_{2}$ gas is often dissolved in normal saline under high pressure to produce $\mathrm{H}_{2}$-rich saline, which exerts similar protective effects (29). Notably, it is safer and more convenient in practice.

The pathophysiological effects of DM are closely associated with oxidative stress (30), and several studies have examined the antidiabetic effects of $\mathrm{H}_{2}$. Kim and $\mathrm{Kim}$ (31) reported that electrolyzed reduced water with ROS scavenging 


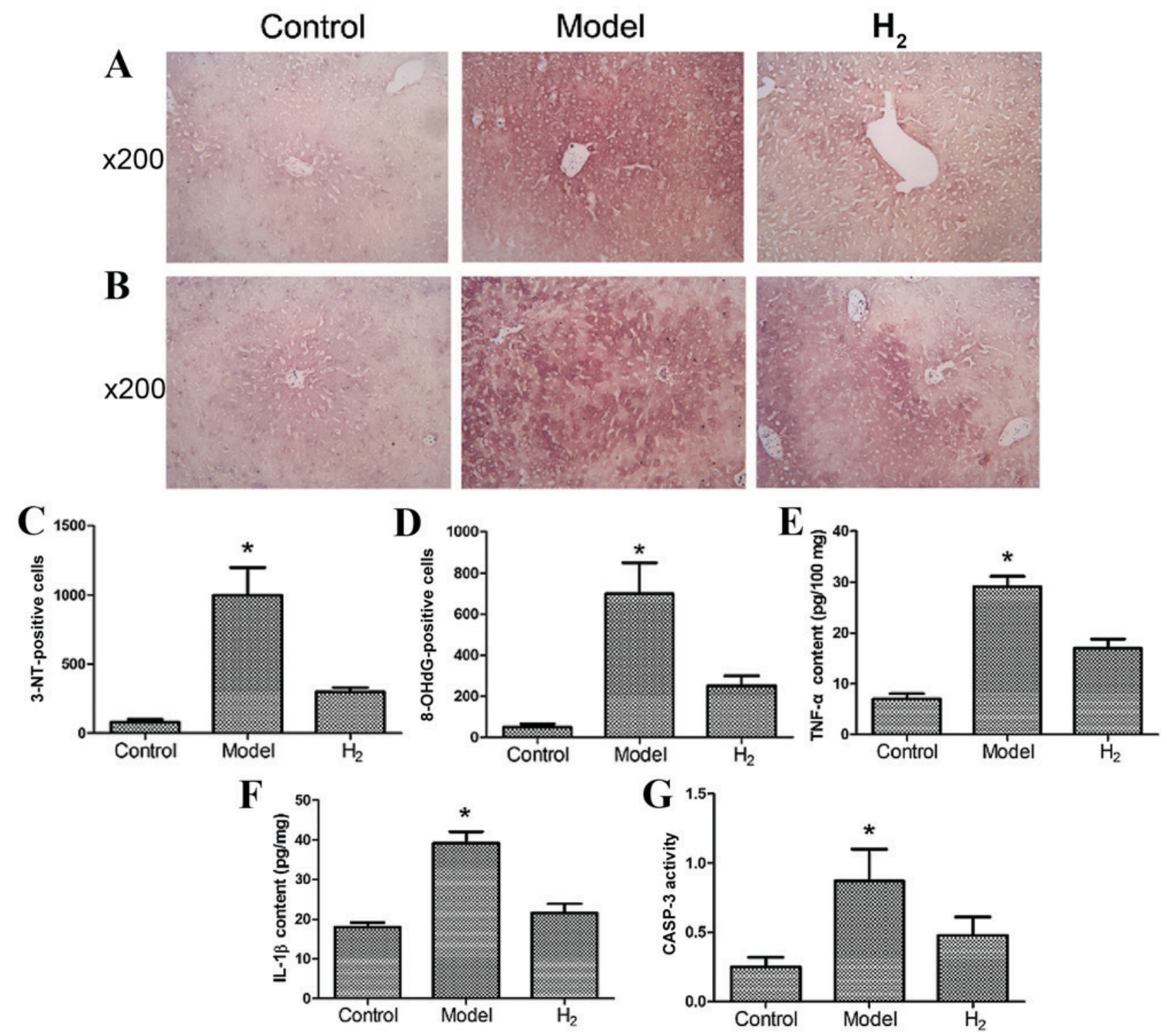

Figure 3. Determination of 3-NT, 8-OHdG, TNF- $\alpha$, IL-1 $\beta$ and CASP-3 levels. Representative immunohistochemical staining of (A) 3-NT and (B) 8-OHdG at x200 original magnification. Number of (C) 3-NT- and (D) 8-OHdG-positive cells. (E) TNF- $\alpha$ and (F) IL-1 $\beta$ content. (G) CASP-3 activity. " P<0.05 vs. control and $\mathrm{H}_{2}$ groups. 3-NT, 3-nitrotyrosine; 8-OHdG, 8-hydroxy-2'-deoxyguanine; CASP-3, caspase-3; $\mathrm{H}_{2}$, molecular hydrogen; IL-1 1 , interleukin-1 beta; TNF- $\alpha$, tumor necrosis factor alpha.

ability had a potential effect on diabetic animals, significantly reducing blood glucose concentration and improving glucose tolerance. Kajiyama et al (12) demonstrated that in patients with $\mathrm{T} 2 \mathrm{DM}$, intake of $\mathrm{H}_{2}$-rich water was associated with significant decreases in the levels of modified low-density lipoprotein (LDL) cholesterol, small dense LDL and urinary 8 -isoprostanes by $15.5,5.7$ and $6.6 \%$, respectively, and concluded that supplementation with $\mathrm{H}_{2}$-rich water may have a beneficial role in the prevention and treatment of T2DM and insulin resistance. Kamimura et al (15) reported that $\mathrm{H}_{2}$ improved obesity and DM by inducing the expression of hepatic fibroblast growth factor 21 (FGF21) and improving energy metabolism in diabetic $d b / d b$ mice, which is an important finding in the study of the mechanism of antioxidative effects of $\mathrm{H}_{2}$. The present study revealed that $\mathrm{H}_{2}$-rich saline ameliorated hepatic oxidative stress, which was demonstrated by a reduction in the levels of TNF- $\alpha$, IL-1 $\beta$, 3-NT and 8-OHdG in the liver.
The three PPAR subtypes (PPAR $\alpha, \operatorname{PPAR} \beta$ and PPAR $\gamma$ ) belong to the nuclear receptor superfamily of transcription factors, and their response elements are located in the promoter region of FGF21. PPAR $\alpha$ and PPAR $\gamma$ are two important upstream regulators of FGF21. PPAR $\alpha$ is predominantly expressed in the liver and regulates lipid metabolism through activating the expression of various proteins, such as lipoprotein lipase and diacylglycerol acyltransferase. PPAR $\gamma$ is mainly found in adipose and hepatic tissue, and its activation serves a major role in increasing insulin sensitivity (32).

The present study demonstrated that $\mathrm{H}_{2}$-rich saline significantly increased the expression levels of PPAR $\alpha$ and PPAR $\gamma$, and significantly improved glucose and lipid metabolism, as demonstrated by reduced serum levels of TC and TG, and improved insulin sensitivity and glucose tolerance. The present study also examined the serum levels of ALT and TBIL. Although $\mathrm{H}_{2}$ lowered the levels of ALT and TBIL, the difference was not statistically significant compared with 

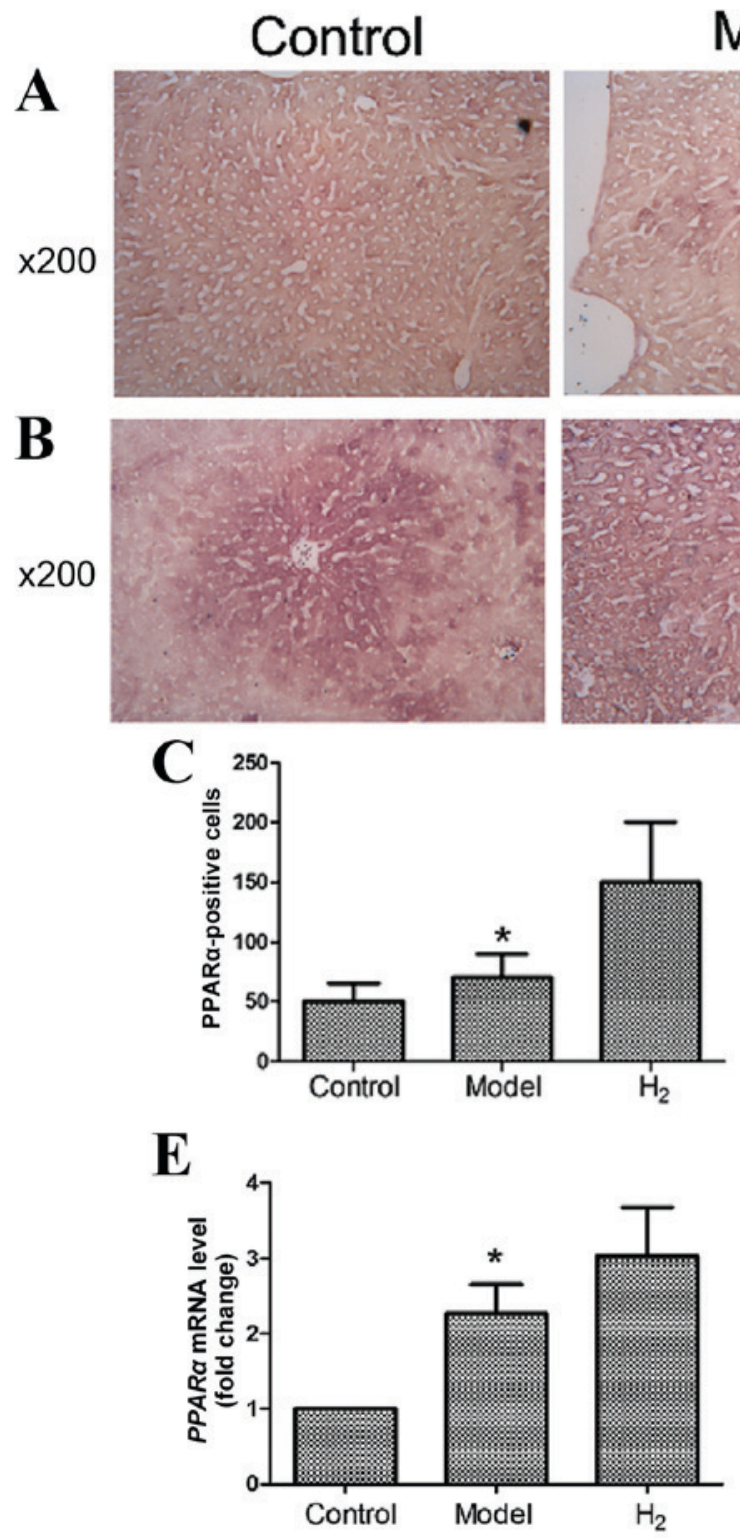

Model
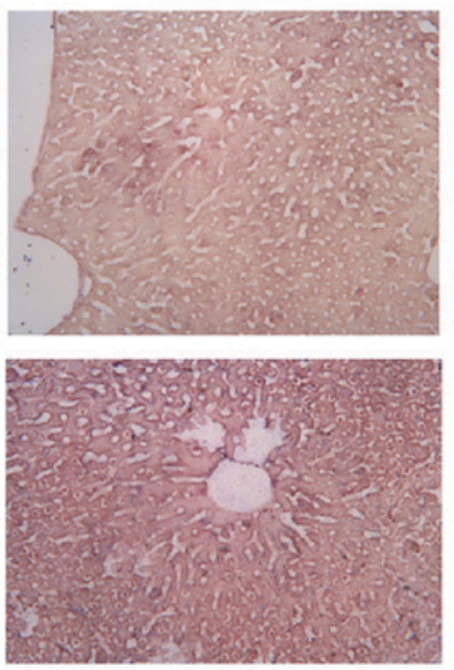

D

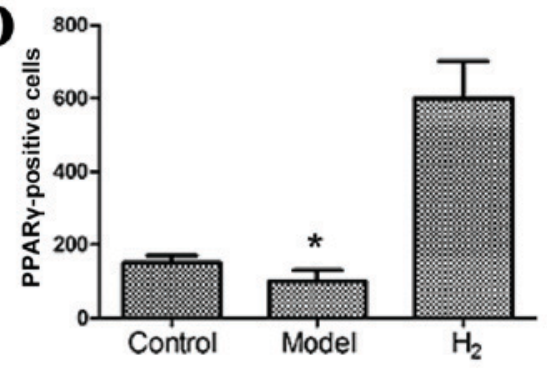

F

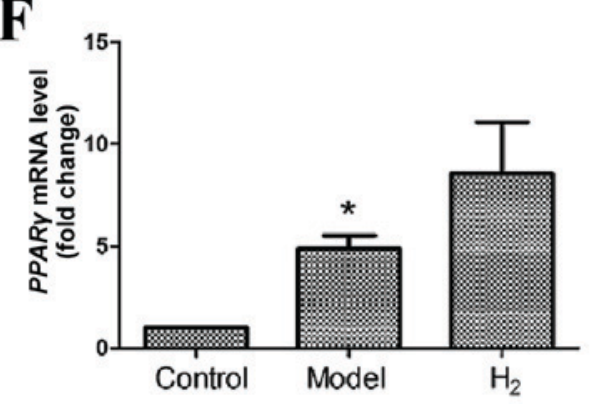

Figure 4. Determination of PPAR $\alpha$ and PPAR $\gamma$. Representative immunohistochemical staining of (A) PPAR $\alpha$ and (B) PPAR $\gamma$ at $x 200$ original magnification. Numbers of (C) PPAR $\alpha$ - and (D) PPAR $\gamma$-positive cells. (E and F) PPAR $\alpha$ and PPAR $\gamma$ mRNA expression levels were quantified by reverse transcription-quantitative polymerase chain reaction. ${ }^{*} \mathrm{P}<0.05$ vs. control and $\mathrm{H}_{2}$ groups. $\mathrm{H}_{2}$, molecular hydrogen; PPAR, peroxisome proliferator-activated receptor.

the model group. The present study attributed this result to the powerful compensatory capacity of the liver and the relatively short experimental time. It is expected that, if the experiment were conducted for a longer period of time and the damages to the liver went beyond the hepatic compensatory ability, liver function may be compromised.

Upon activation, PPAR $\alpha$ and PPAR $\gamma$ synergistically inhibit fatty acid synthesis by regulating the expression of sterol regulatory element-binding protein-1c and its target gene fatty acid synthase (33). PPAR $\alpha$ and PPAR $\gamma$ activation exerts anti-inflammatory effects on the liver by reducing hepatic steatosis, downregulating the expression of inflammatory genes and attenuating inflammation in adipose tissue (34). Furthermore, PPAR $\alpha$ and PPAR $\gamma$ could regulate the production of free radicals by increasing the expression of superoxide dismutase and reducing NADPH oxidase activity $(35,36)$.
In conclusion, the protective effects of $\mathrm{H}_{2}$ on high-sugar and high-fat diet-induced NAFLD may be attributed to its direct antioxidative properties, as well as its activation of PPAR $\alpha$ and PPAR $\gamma$. With regards to PPAR activation, $\mathrm{H}_{2}$-rich saline shares the same mechanism as rosiglitazone, which is a potent PPAR $\gamma$ receptor activator. Although further studies are required, $\mathrm{H}_{2}$-rich saline may be considered a potential agent in the prevention and treatment of NAFLD.

\section{Acknowledgements}

The authors would like to thank Professor Yan Zhang from the Department of Foreign Language Teaching of the Second Military Medical University for her assistance with the grammar and readability of the manuscript. This study is supported by the Changhai 1255 Scientific Research Project (grant no. CH125541900). 


\section{References}

1. Adams LA, Lymp JF, St Sauver J, Sanderson SO, Lindor KD, Feldstein A and Angulo P: The natural history of nonalcoholic fatty liver disease: A population-based cohort study. Gastroenterology 129: 113-121, 2005.

2. Angulo P: Nonalcoholic fatty liver disease. N Engl J Med 346: $1221-1231,2002$

3. Clark JM: The epidemiology of nonalcoholic fatty liver disease in adults. J Clin Gastroenterol 40 (Suppl 1): S5-S10, 2006.

4. Ekstedt M, Franzén LE, Mathiesen UL, Thorelius L, Holmqvist M, Bodemar G and Kechagias S: Long-term follow-up of patients with NAFLD and elevated liver enzymes. Hepatology 44: 865-873, 2006.

5. Porepa L, Ray JG, Sanchez-Romeu P and Booth GL: Newly diagnosed diabetes mellitus as a risk factor for serious liver disease. CMAJ 182: E526-E531, 2010.

6. Giacco F and Brownlee M: Oxidative stress and diabetic complications. Circ Res 107: 1058-1070, 2010.

7. Ohsawa I, Ishikawa M, Takahashi K, Watanabe M, Nishimaki K, Yamagata K, Katsura K, Katayama Y, Asoh S and Ohta S: Hydrogen acts as a therapeutic antioxidant by selectively reducing cytotoxic oxygen radicals. Nat Med 13: 688-694, 2007.

8. Hayashida K, Sano M, Ohsawa I, Shinmura K, Tamaki K, Kimura K, Endo J, Katayama T, Kawamura A, Kohsaka S, et al: Inhalation of hydrogen gas reduces infarct size in the rat model of myocardial ischemia-reperfusion injury. Biochem Biophys Res Commun 373: 30-35, 2008.

9. Fukuda KI, Asoh S, Ishikawa M, Yamamoto Y, Ohsawa I and Ohta S: Inhalation of hydrogen gas suppresses hepatic injury caused by ischemia/reperfusion through reducing oxidative stress. Biochem Biophys Res Commun 361: 670-674, 2007.

10. Buchholz BM, Kaczorowski DJ, Sugimoto R, Yang R, Wang Y, Billiar TR, McCurry KR, Bauer AJ and Nakao A: Hydrogen inhalation ameliorates oxidative stress in transplantation induced intestinal graft injury. Am J Transplant 8: 2015-2024, 2008.

11. Ohsawa I, Nishimaki K, Yamagata K, Ishikawa M and Ohta S: Consumption of hydrogen water prevents atherosclerosis in apolipoprotein E knockout mice. Biochem Biophys Res Commun 377: 1195-1198, 2008.

12. Kajiyama S, Hasegawa G, Asano M, Hosoda H, Fukui M, Nakamura N, Kitawaki J, Imai S, Nakano K, Ohta M, et al: Supplementation of hydrogen-rich water improves lipid and glucose metabolism in patients with type 2 diabetes or impaired glucose tolerance. Nutr Res 28: 137-143, 2008.

13. Sato Y, Kajiyama S, Amano A, Kondo Y, Sasaki T, Handa S, Takahashi R, Fukui M, Hasegawa G, Nakamura N, et al: Hydrogen-rich pure water prevents superoxide formation in brain slices of vitamin C-depleted SMP30/GNL knockout mice. Biochem Biophys Res Commun 375: 346-350, 2008.

14. Fu Y, Ito M, Fujita Y, Ito M, Ichihara M, Masuda A, Suzuki Y, Maesawa S, Kajita Y, Hirayama M, et al: Molecular hydrogen is protective against 6-hydroxydopamine-induced nigrostriatal degeneration in a rat model of Parkinson's disease. Neurosci Lett 453: 81-85, 2009.

15. Kamimura N, Nishimaki K, Ohsawa I and Ohta S: Molecular hydrogen improves obesity and diabetes by inducing hepatic FGF2 1 and stimulating energy metabolism in $\mathrm{db} / \mathrm{db}$ mice. Obesity (Silver Spring) 19: 1396-1403, 2011.

16. Garber J, Barbee R, Bielitzki J, Clayton L, Donovan J, Hendriksen C, Kohn D, Lipman N, Locke P, Melcher J, et al: Guide for the Care and Use of Laboratory Animals. 8th edition. Washington (DC), National Academies Press (US), 2011.

17. Li L, Chen L, Hu L, Liu Y, Sun HY, Tang J, Hou YJ, Chang YX, Tu QQ, Feng GS, et al: Nuclear factor high-mobility group box 1 mediating the activation of Toll-like receptor 4 signaling in hepatocytes in the early stage of nonalcoholic fatty liver disease in mice. Hepatology 54: 1620-1630, 2011.

18. Nugent DA, Smith DM and Jones HB: A review of islet of Langerhans degeneration in rodent models of type 2 diabetes. Toxicol Pathol 36: 529-551, 2008.
19. Agwaya MS, Vuzi PC and Nandutu AM: Hypoglycemic activity of aqueous root bark extract zanthoxylum chalybeum in alloxan-induced diabetic rats. J Diabetes Res 2016: 8727590, 2016.

20. De Oliveira JC, Ludemann Camargo R, Barella LF, Chaves Souto Branco R, Gravena C, Grassiolli S, Torrezan R and Cezar De Freitas Mathias P: Anesthetic-induced transient hyperglycemia and insulin resistance do not depend on the sympathoadrenal axis. Minerva Endocrinol 38: 379-388, 2013.

21. Shi J, Yao F, Zhong C, Pan X, Yang Y and Lin Q: Hydrogen saline is protective for acute lung ischaemia/reperfusion injuries in rats. Heart Lung Circ 21: 556-563, 2012.

22. Brunt EM, Janney CG, Di Bisceglie AM, Neuschwander-Tetri BA and Bacon BR: Nonalcoholic steatohepatitis: A proposal for grading and staging the histological lesions. Am J Gastroenterol 94: 2467-2474, 1999.

23. Knodell RG, Ishak KG,Black WC, Chen TS, Craig R, Kaplowitz N, Kiernan TW and Wollman J: Formulation and application of a numerical scoring system for assessing histological activity in asymptomatic chronic active hepatitis. Hepatology 1: 431-435, 1981.

24. Lee GS, Yan JS, Ng RK, Kakar S and Maher JJ: Polyunsaturated fat in the methionine-choline-deficient diet influences hepatic inflammation but not hepatocellular injury. J Lipid Res 48: 1885-1896, 2007.

25. Zhai X, Chen X, Shi J, Shi D, Ye Z, Liu W, Li M, Wang Q, Kang Z, Bi H and Sun X: Lactulose ameliorates cerebral ischemia-reperfusion injury in rats by inducing hydrogen by activating Nrf2 expression. Free Radic Biol Med 65: 731-741, 2013.

26. Livak KJ and Schmittgen TD: Analysis of relative gene expression data using real-time quantitative PCR and the 2(-Delta Delta C (T)) Method. Methods 25: 402-408, 2001.

27. Day CP and James OF: Steatohepatitis: A tale of two 'hits'? Gastroenterology 114: 842-845, 1998.

28. Leite NC, Villela-Nogueira CA, Cardoso CR and Salles GF: Non-alcoholic fatty liver disease and diabetes: From physiopathological interplay to diagnosis and treatment. World $\mathrm{J}$ Gastroenterol 20: 8377-8392, 2014.

29. Cai J, Kang Z, Liu K, Liu W, Li R, Zhang JH, Luo X and Sun X: Neuroprotective effects of hydrogen saline in neonatal hypoxia-ischemia rat model. Brain Res 1256: 129-137, 2009.

30. Suzuki H, Kayama Y, Sakamoto M, Iuchi H, Shimizu I, Yoshino T, Katoh D, Nagoshi T, Tojo K, Minamino T, et al: Arachidonate 12/15-lipoxygenase-induced inflammation and oxidative stress are involved in the development of Diabetic Cardiomyopathy. Diabetes 64: 618-630, 2015

31. Kim MJ and Kim HK: Anti-diabetic effects of electrolyzed reduced water in streptozotocin-induced and genetic diabetic mice. Life Sci 79: 2288-2292, 2006.

32. Zhao X, Xue J, Wang XL, Zhang Y, Deng M and Xie ML: Involvement of hepatic peroxisome proliferator-activated receptor $\alpha / \gamma$ in the therapeutic effect of osthole on high-fat and high-sucrose-induced steatohepatitis in rats. Int Immunopharmacol 22: 176-181, 2014.

33. Konig B, Koch A, Spielmann J, Hilgenfeld C, Hirche F, Stangl GI and Eder K: Activation of PPARalpha and PPARgamma reduces triacylglycerol synthesis in rat hepatoma cells by reduction of nuclear SREBP-1. Eur J Pharmacol 605: 23-30, 2009.

34. Stienstra R, Mandard S, Patsouris D, Maass C, Kersten S and Muller M: Peroxisome proliferator-activated receptor alpha protects against obesity-induced hepatic inflammation. Endocrinology 148: 2753-2763, 2007.

35. Kim JC, Lee YH, Yu MK, Lee NH, Park JD, Bhattarai G and Yi HK: Anti-inflammatory mechanism of PPAR $\gamma$ on LPS-induced pulp cells: Role of the ROS removal activity. Arch Oral Biol 57: 392-400, 2012.

36. Inoue I, Goto S, Matsunaga T, Nakajima T, Awata T, Hokari S, Komoda $\mathrm{T}$ and Katayama S: The ligands/activators for peroxisome proliferator-activated receptor alpha (PPARalpha) and PPARgamma increase $\mathrm{Cu} 2+, \mathrm{Zn} 2+$-superoxide dismutase and decrease $\mathrm{p} 22$ phox message expressions in primary endothelial cells. Metabolism 50: 3-11, 2001. 